\title{
Knowledge and Proficiency in Skills of Initial Trauma Management among Healthcare Personnel in Secondary Care
}

\author{
Embu HY, ${ }^{1}$ Nuhu SI, ${ }^{2}$ Bishmang SP. ${ }^{3}$ \\ 'Department of Anaesthesia, Jos University Teaching Hospital, Jos, Plateau state. Nigeria. \\ ${ }^{2}$ Department of Anaesthesia, Jos University Teaching Hospital, Jos, Plateau state. Nigeria. \\ ${ }^{3}$ Plateau State Specialist Hospital, Jos, Plateau state. Nigeria.
}

*Corresponding Author: Dr. Henry Y. Embu. Department of Anaesthesia, Jos University Teaching Hospital, P.M.B. 2076 Jos.

E-amail:embuy@yahoo.com.

\begin{abstract} Date Published: 03/04/2020

Advanced Trauma Life Support (ATLS) protocols aim to provide good trauma care by enhancing the skills of medical personnel all over the world and while this is well established in developed countries it does not appear to be so in developing countries. This study aims to assess the knowledge and proficiency in initial trauma management skills among health caregivers in some general/cottage hospitals in north central Nigeria. Questionnaires were developed to assess the knowledge and skills of caregivers in airway management, respiratory distress and shock. The questionnaires were administered on caregivers involved in trauma care in some general/cottage hospitals that offer secondary care in a state in north-central Nigeria. There were 34 health workers who responded, 10 (29.41\%) were doctors and 24(70.54\%) were nurses. Their years of experience were from 2 to 35 years (median 14years). Ten $(29.41 \%$ ) had at least one training in ATLS in the past while $24(70.59 \%)$ had none. In assessing their management skills, $97.06 \%$ reported they were able to assess the airway, $88.24 \%$ could do chin lift, $73.53 \%$ jaw thrust while $91.18 \%$ were able to insert oral airway. Thirty-one $(91.18 \%)$ were able to recognize respiratory distress, 88.24 were able to administer oxygen using facemask and $64.71 \%$ using nasal prong. Thirty (88.24\%) could assess a patient for shock, $82.35 \%$ could splint fractures for haemorrhage control. Twenty-seven (79.41\%) reported knowing parameters to monitor during resuscitation. We concluded that knowledge of airway management was high but proficiency and confidence in performing these skills were low.
\end{abstract} Date Accepted: $05 / 03 / 2020$

Keywords: Initial trauma management, knowledge,Secondary care

\section{INTRODUCTION}

\begin{abstract}
A frica bears a heavy burden from trauma with more deaths occurring each year from injury than deaths linked to HIV/AIDS, tuberculosis and malaria combined. ${ }^{1}$ Among the various aetiologies of trauma, the most prevalent is road traffic crashes. It has been reported that though developing countries account for only $48 \%$ of
\end{abstract}

motorized vehicles they however account for $91 \%$ of fatalities of road traffic crashes per annum. ${ }^{2}$ In Nigeria like in many developing countries road traffic crashes is a major public health issue. According to the Federal Road Safety Corp (FRSC) Nigeria is ranked 191 out of 192 countries with the deadliest roads and road traffic deaths 
account for $1.46 \%$ of all deaths in the country. ${ }^{3}$ It has been reported that at least a third of hospital trauma deaths could be prevented with optimal response from the emergency health system. ${ }^{4}$ The knowledge and skills of the healthcare providers forms an important integral part of the optimal response to trauma patients in our hospitals.

Advanced trauma life support (ATLS) has revolutionalized in-hospital management of major trauma patients and is now accepted as a standard in many countries worldwide. ${ }^{5}$ The ATLS protocol has been found to enhance the knowledge and skills of healthcare personnel and while this is well established in developed countries, it is not so in many developing countries like Nigeria.

A great number of trauma incidences in Nigeria occur in rural communities. Since pre-hospital care is non-existent the first contact many of the victims have with healthcare givers is often at the primary and secondary health facilities. Our study is designed to assess the knowledge and proficiency in the skills of resuscitating trauma patients among healthcare personnel in some general and cottage hospitals that provide secondary healthcare in Plateau state, North-central Nigeria.

\section{MATERIALSAND METHODS}

A semi-structured questionnaire was developed to assess the knowledge and skills of healthcare givers in airway assessment and management and also assessment and management of respiratory distress and shock. The skills assessed were as recommended by the 'Guidelines for Essential Trauma Care' for the level of care that corresponds to secondary care in our setting. The guidelines were drawn up by a number of bodies which include; WHO, International Association for surgery of trauma and surgical intensive care and International Society of Surgery. ${ }^{6}$

In the guidelines each skill could belong to one of three categories for the specific level of care;

- Essential (E) means that the skill should be assured for the stated level of healthcare system in all cases

- Desirable (D) represents capability that increases the probability of a successful outcome of trauma care

- Irrelevant (I) implies that one would not expect this capability at that level of healthcare system even with full availability of resources

The skills/capabilities used to design the questionnaire were those considered 'Essential' for secondary healthcare providers by the guidelines.
The questionnaires were administered to doctors and nurses directly involved in the care of trauma patients in some government owned general/ cottage hospitals that offered secondary care in Plateau state. These were personnel who worked at the emergency departments of their respective hospitals. The questionnaires were administered between $4^{\text {th }}$ and $25^{\text {th }}$ of June 2015 and sought to know if the respondents were able to perform the stated procedures. It also asked about their level of proficiency as a mark of confidence level. The level of proficiency was measured on a scale of four; 'not proficient', 'somewhat proficient', 'proficient' and 'very proficient'. Those who reported being not proficient and somewhat proficient were considered not proficient/ confident in carrying the stated procedures while those who reported being proficient or very proficient were considered proficient/ confident in carrying out the procedures.

\section{Data Analysis}

Data was analyzed using Epi info version 7 statistical package. Categorical data was presented as frequency and percentage while continuous data were presented as mean and standard deviation. Statistical significance was defined as $\mathrm{P}$ value less than 0.05 .

\section{RESULTS}

Out of 55 questionnaires that were sent out 34 were returned complete. That was $61.8 \%$ response from eight out of the 14 public secondary healthcare facilities in the state. Ten (29.41\%) of the respondents were doctors and 24 (70.59\%) were nurses. The male to female ratio was 3.25:1

The respondents' years of experience was between 2 years and 35 years (median 14 years). Ten $(29.41 \%)$ have had at least one training in ATLS or its equivalent since qualification while $24(70.59 \%)$ did not have any training in ATLS. Eight $(80 \%)$ of those who had training in ATLS were doctors while $2(20 \%)$ were nurses

In assessing their airway management skills,33(97.06\%) said they were able to carry out airway assessment,30(88.24\%) were able to perform chin lift,25(73.53\%) were able to perform jaw thrust, $31(91.18 \%)$ were able to insert an oral airway and 23(67.66\%) a nasal airway. Table 1 .

Concerning management of breathing, 31(91.18\%) respondents were able to recognize respiratory distress,30(88.24\%) were able to administer oxygen using facemask and 22(64.71\%) using nasal catheter/prongs. Ten 
respondents i.e. $29.41 \%$ were able to perform a needle thoracostomy and 6(17.65\%) pass a chest tube. Table 2.

In the management of circulation, 30(88.24\%) respondents could assess a patient in shock, 28(82.35\%) could splint fractures for haemorrhage control and $3(8.82 \%)$ could perform a pelvic wrap for haemorrhage control while $8(23.53 \%)$ were able to do a peripheral venous cut down. None could access an intraosseous line. Table 3.

When asked if they knew the parameters that should be monitored during resuscitation, 27(79.41\%) said they knew the parameters to monitor during resuscitation. When asked to mention the parameters; $19(55.88 \%)$ mentioned blood pressure, 18(52.29\%) mentioned pulse rate, 11(32.35\%) mentioned respiratory rate, $7(20.59 \%)$ mentioned temperature, 2(5.88\%) mentioned urine output and oxygen saturation respectively and $1(2.94 \%)$ mentioned central venous pressure (CVP).

Twenty four $(70.59 \%)$ reported either not being or being somewhat proficient in at least one of the manouvres while $8(23.53 \%)$ reported being proficient and $2(5.88 \%)$ very

\section{Table 1. Airway management skills of respondents}

\begin{tabular}{lc}
\hline Procedure & $\begin{array}{c}\text { Respondents able to carry out procedure } \\
\text { n (\%) }\end{array}$ \\
\hline Assessment of airway compromise & $33(97.06)$ \\
Chin lift & $30(88.24)$ \\
Jaw thrust & $25(73.53)$ \\
Recovery position & $33(97.06)$ \\
Insertion of oral airway & $31(91.18)$ \\
Insertion of nasalairway & $23(67.63)$ \\
Use of suction & $34(100)$ \\
\hline
\end{tabular}

Table 2. Skills for the management of respiratory problems in trauma victims

\begin{tabular}{lc}
\hline Procedure & $\begin{array}{c}\text { Respondents able to carry out the } \\
\text { procedure } n(\%)\end{array}$ \\
\hline Assessment of respiriatory distress and adequacy of ventilation & $31(91.18)$ \\
Administrtation of oxygen using face mask & $30(88.24)$ \\
Bag mask ventilation & $15(44.12)$ \\
Administrtation of oxygen using nasal catheterl prongs & $22(64.71)$ \\
Needle thoracostomy & $10(29.41)$ \\
Chest tube insertion & $6(17.65)$ \\
\hline
\end{tabular}

\begin{tabular}{ll|ll|ll|l} 
J Biomed Res. Clin Pract $\mid$ Vol 3 & No & 1 & 2020
\end{tabular}
Table 3. Skills in the management of circulatory problems in trauma victims

\begin{tabular}{lc}
\hline s/no Procedure & $\begin{array}{l}\text { Respondents who are able to carry out } \\
\text { the procedure n(\%) }\end{array}$ \\
\hline Assessment of shock & $30(88.24)$ \\
Arterial tourniquet & $29(85.29)$ \\
Splinting of fractures for haemorrhage control & $28(82.35)$ \\
Pelvic wrap for haemorrhage control & $3(8.82)$ \\
Fluid resuscitation & $30(88.24)$ \\
Peripheral venous access & $33(97.06)$ \\
Peripheral venous cut down access & $8(23.53)$ \\
Transfusion knowledge & $31(91.18)$ \\
Intraosseous access & $0(0)$ \\
\hline
\end{tabular}

proficient in one or more manouvres. Those who reported being either non proficient or somewhat proficient were considered not confident while those who report to be proficient or very proficient were considered confident in carrying out the procedures. When the level of confidence was compared among those who had ATLS in the past and those who did not, it was found that confidence was higher among those who had ATLS in the past (70\% vs $4.2 \%) \mathrm{p}=$ 0.019 .

\section{DISCUSSION}

Even though the evidence shows that a significant percentage of trauma deaths occur in the pre-hospital setting ${ }^{7,8}$ and that many deaths could be prevented by proper measures, ${ }^{9,10}$ prehospital trauma care is mostly non-existent in Nigeria. Our study though attempts to look at whether survivors who make it to public secondary health care facilities in this part of the country are likely to get optimum care.

The number of our respondents who had undergone training in trauma care like the Advanced Trauma Life Support (ATLS) or its equivalent post qualification was low. Though a significant number of our respondents said they were able to perform basic airway manouvres their ability to administer oxygen using basic devices was not encouraging and assessment of the cardiovascular system and interventions to arrest haemorrhage were poor.

Much of the improvement in trauma patient outcome in highincome countries has come from improvements in the organization of trauma care services and it is true that improvements in the organization of trauma care services can 
be achieved in almost every setting including low-income countries. ${ }^{11}$ Instances abound in low-income countries where improvements in organization of trauma care had led to greatly improved outcomes. ${ }^{12}$ These may be in the form of better policies, infrastructure and training. Improvements in Cambodia, Iraq and Mexico primarily emphasized training. ${ }^{12}$ The training may range from short courses or workshops lasting a few days to those that last months for full certification.

Wong, ${ }^{13}$ in a study among basic surgical trainees in Australia with a mean length of time post graduation of 2.5 years found that only $14 \%$ had completed training in ATLS. The average years post qualification among our respondents was 14 years and $29.41 \%$ had undergone at least one training in ATLS or its equivalent post qualification. None of the training was in the previous one year. ATLS is the most widely utilized trauma course world wide and in Nigeria, trauma courses like the Jos trauma course by the Jos University Teaching Hospital are mostly adapted from ATLS protocols. The ATLS course is conducted in Nigeria by organizations like the International Committee of the Red Cross (ICRC). The course usually lasts two to three days and is oriented primarily towards the first few hours of care in an emergency department.

Trauma training should involve medical and nursing staff who care for the injured. The hospitals from which we got responses were rural based and the doctors were general practitioners. Some of the hospitals had only one doctor who may not always be on ground. While not all the doctors who responded had undergone training in ATLS or its equivalent, very few of the nurses had the training and these training are known to improve trauma care in a variety of environments including developing countries..$^{14,15}$

With respect to the management of the airway $97.06 \%$ could assess the airway. Airway management is part of the ABCDE system of approach to resuscitation of major trauma victims as outlined in ATLS. It is important that healthcare personnel involved in the resuscitation of trauma victims are knowledgeable in recognizing airway obstruction and skillful in applying manouvres to open up the airway. Our questionnaire inquired about simple manouvres like the application of chin lift, jaw thrust and insertion of oropharyngeal airway which are considered essential skills for personnel at the secondary level of healthcare. In the study by Wong, ${ }^{13} 63 \%$ of respondents reported being confident in managing the airway.

A good number of our respondents reported being able to recognize respiratory distress and $88.24 \%$ were able to administer oxygen using face mask but only $64.71 \%$ could administer oxygen using nasal catheter or prongs. These are very simple procedures to carry out which can easily be learnt but have very great impact on the care of trauma patients with respiratory compromise. Only $29.41 \%$ of our respondents were able to perform needle thoracostomy and $17.65 \%$ pass a chest tube. This is comparable to the outcome in the Wong study which reported that $20 \%$ of his respondents were confident in inserting a chest drain but then, his respondents were junior doctors with an average of 2.5 years post graduation while our respondents were more experienced with an average of 14 years post qualification. All our respondents who reported being able to pass a chest tube were doctors.

The ability to assess a patient for shock is essential for all healthcare personnel taking part in the resuscitation of trauma victims. This often requires simple equipment like a sphygmomanometer and stethoscope but the care giver should be able to assess the pulse, check capillary refill and skin temperature. The respondents' knowledge of the recognition and management of circulatory compromise was not as good as that of airway compromise. Healthcare personnel at this level should possess the ability to control external haemorrhage through manual pressure and pressure dressing. Insertion of central lines is a skill that may often not be achievable at the secondary level care in our country but when access to peripheral veins is not successful other routes for access to the circulation like intraosseous lines and venous cut down should be achievable. Our respondents however had poor knowledge of these routes as a matter of fact none could access an intraosseous line.

Monitoring enables the caregiver to determine whether the care given is achieving the desired results. Knowing the parameters to monitor requires an understanding of the changes that occur during airway compromise and shock. While $79.41 \%$ of our respondents mentioned blood pressure, only $5.88 \%$ mentioned urine output as a parameter to monitor during shock. Despite the central role pulse oximetry plays in monitoring airway and breathing only $5.88 \%$ of respondents mentioned saturation as a parameter to monitor.

Confidence level in performing the procedures was generally low. This was probably because many of the respondents had never performed some of the procedures. This was the findings in some studies where most respondents never performed many of the procedures required during resuscitation. $^{13,16}$ 
A limitation of our study is that it is difficult to gauge knowledge and proficiency in a skill when one has not observed the subject carrying out the stated procedure. Our study was questionnaire based and did not practically assess these skills but relied mainly on the responses by participants. The study involved staff of a few general hospitals and our findings may not be a reflection of what is generally obtainable in the state or region.

\section{CONCLUSION}

We found that on the average the respondents' knowledge and skills in airway assessment and procedures was good for their level of care but proficiency and confidence in performing the procedures was low. The knowledge and confidence progressively decreased from airway assessment and management to the assessment and management of respiration and circulation. Confidence level was significantly higher among participants who had training in ATLS in the past. Training and retraining doctors and nurses involved in trauma care is important and has been shown to cause positive change in practice and improved trauma care when applied to low and middle income countries. ${ }^{17,18,19}$ Regular drills are also important in improving skill, confidence and readiness to manage trauma patients.

\section{Conflicts of Interest}

The authors declare no conflicts of interest.

\section{REFERENCE}

1. WHO. Global burden of Disease - Update 2004: $2014 \mathrm{http} / / \mathrm{www}$. who.int/healthinfo/global-burdendisease/2004_report-update/en/Accessed $16^{\text {th }}$ August 2017

2. Schmucker U, Steifert J, Stengel D, Matthes G, Ottersbach C, Ekkemkemp A. Road traffic crashes in Developing Countries. Unfallchirurg. 2010;113: 373-377. http//dxdoi.org/10.1007/50013-010-1777-9 Accessed 24 $4^{\text {th }}$ September 2017

3. Federal Road Safety Commission (FRSC) Nigeria. Road Traffic Crashes Data. Abuja: FRSC Nigeria. 2010

4. Saleh M. commission on the provision of surgical services: The management of patients with major injuries. Ann R Coll Surg Engl. 1989;58-62

5. Thies K, Gwinnutt C, Driscoll P, Carneiro A, Gomes E, Araujo R et al. The European trauma Course-from concept to course. Resuscitation. 2007;74(1):135-141
6. Guidelines for essential trauma care. WHO, IATSIC, INTERNATIONAL SOCIETY OF SURGERY. 2004;5:19-58

7. Dean D, Wetael B, White N, Kappermann N, Wang NE, Haukoos JS et al. From 9-1-1 call to death: evaluating traumatic deaths in seven regions for early recognition of high-risk patients. J Trauma Acute Care Surg. 2014;76:846-853

8. Bakke H, Wisborg T. Rural high north: a high rate of fatal injury and pre-hospital death. World J Surg. 2011;35:1615-1620

9. Taanvik D, Bakke HK, Wisborg T. A systematic literature review on first aid provided by people to trauma victims. Acta Anaesthesiol Scand. 2012;56:1222-1227

10. Oliver GJ, Walter DP, Redmond AD. Are prehospital deaths from trauma and acci8dental injury preventable? A direct historical comparison to assess what has changed in two decades. Injury Int J Care Injured. 2017;48:978-984

11. Nathens AB, Jurkovich GJ, Cummings P, Rivara FP, Maier RV. The effect of organized systems of trauma care on motor vehicle crash mortality. JAMA. 2000;283:1990-1994

12. WHO. Strengthening care for the injured: success stories and lessons learned from around the world. 2010. www.who.int/violence_injury_prevention. Accessed 20th August, 2018

13. Wong K. Trauma education of junior hospital doctors at a major Australian trauma service. Surgeon. 2007;5(2):76-80

14. Mock CN, Quansah RE, Addae-Mensah L. Kwame Nkrumah University of Science and Technology continuing medical education course in trauma management. Trauma Quarterly. 1999;14(3):345-348

15. Quansah R. Availability of emergency medical services along major highways. Ghana Medical Journal. 2001;35:8-10

16. Espisito TJ, Coposs MK, Maler RV. Analysis of surgical participation of the Advanced Trauma Life Support course. What are the goals and are we meeting them? Arch Surg. 1992;127(6):721-725

17. Nguyen TS, Mock CN. Assessment of the status of essential trauma care in Hanoi and Khan Hoa, Vietnam. Injury. 2007;38:1014-1022

18. Van Olden GD, Meeuwis JD, Bolhus HW, Boxma H, Goris RJ. Clinical impact of Advanced Trauma Life Support. Am J Emerg Med. 2004;22(7):522-525

19. Ologunde R, Le G, Turner J, Pandit H, Peter N, Maurer D et al. do trauma courses change practice? A qualitative review of 20 courses in East, Central and Southern Africa. Injury. 2017. DOI.http//dx.doi.org/10.10.16/j.injury.2017.06.007 Accessed $18^{\text {th }}$ August, 2018. 